\title{
Clinical Aspects and Prognostic Factors for Survival in Patients with Recurrent Cervical Cancer after Radical Hysterectomy and Adjuvant Radiochemotherapy
}

\section{Huiting Zhu}

Liaoning Provincial Tumor Hospital: Liaoning Cancer Institute and Hospital https://orcid.org/00000002-4335-9537

\section{Wenjuan Yan}

Medical School of Nanging University

Yuhua Gao ( $52437438 @ q q . c o m$ )

Liaoning Provincial Tumor Hospital: Liaoning Cancer Institute and Hospital

\section{Research Article}

Keywords: Recurrent cervical cancer , Prognostic factors, Survival , Radical surgery , Radiotherapy , Chemotherapy

Posted Date: May 28th, 2021

DOl: https://doi.org/10.21203/rs.3.rs-461567/v1

License: (a) (i) This work is licensed under a Creative Commons Attribution 4.0 International License.

Read Full License 
Clinical Aspects and Prognostic Factors for Survival in Patients with

Recurrent Cervical Cancer after Radical Hysterectomy and

Adjuvant Radiochemotherapy

First author: Zhu Huiting, Department of Gynecologic Oncology, Liaoning Provincial Tumor Hospital, Shenyang 110041, Liaoning, China ORCID iD: https://orcid.org/0000-0002-4335-9537

Second author: Yan Wenjuan, Research Institute of General Surgery, Jinling Hospital, Medical School of Nanging University, Nanjing 210002, Jiangsu, China

Corresponding author: Gao Yuhua, Department of Gynecologic Oncology, Liaoning Provincial Tumor Hospital, Shenyang 110041, Liaoning, China E-mail: 52437438@qq.com 


\begin{abstract}
Purpose To investigate the recurrence patterns and prognostic factors of patients with recurrent cervical cancer after radical hysterectomy with node dissection (RHND) followed by adjuvant radiotherapy (RT)/concurrent radiochemotherapy (CCRT).
\end{abstract}

Methods Between January 1, 2012 and May 31, 2018, the medical records of 153 patients with pre-operative FIGO stage IB-IIA disease treated with RHND followed by adjuvant RT/CCRT in Liaoning Cancer Hospital were retrospectively analyzed.

Results The median disease progression-free survival (PFS) time was 16 months. 75.2\% (115/153) patients had disease relapse within 2 years. The survival of patients with recurrences in multiple organs was signifificantly lower in comparison to those with recurrences in single organ $(P<0.001)$. The survival rate of patients with distant metastasis (DM) and distant metastasis with local recurrence (LR) was significantly lower than that of patients with simple LR $(P=0.006, P<0.001)$. Furthermore, the survival rate of patients with LR+DM was significantly lower than that of patients with simple DM ( $P=0.046)$. The multivariate analysis showed that resection margin involvement, para-aortic and common iliac lymph node metastasis, DM, no treatment after disease relapse and early disease relapse were independent prognostic fators asscociated with poor survivals.

Conclusion Most cervical cancer patients who received initial RHND followed by adjuvant RT/CCRT occurred disease relapse within 2 years. Resection margin involvement, para-aortic and common iliac lymph node metastasis, DM, no treatment after recurrence and early disease relapse were found to be prognostic factors in patients with recurrent cervical cancer after RHND followed by adjuvant RT/CCRT.

Keyword Recurrent cervical cancer $\cdot$ Prognostic factors $\cdot$ Survival $\cdot$ Radical surgery $\cdot$ Radiotherapy $\cdot$ Chemotherapy

\title{
Introduction
}

Cytological screening has made the incidence rate and mortality rate of cervical cancer significantly reduced, but cervical cancer is still the fourth most common malignancy in women [1-2]. When cervical cancer patients detected at early stage (stages IB-IIA) based on the 2009 International Federation of Gynecology and Obstetrics (FIGO) staging system, radical hysterectomy with node dissection (RHND) is the preferred surgical treatment. Postoperative adjuvant radiotherapy (RT) or concurrent chemoradiotherapy (CCRT) is recommended according to the risk factors on postoperative histopathological examination [3-4].

Intermediate-risk factors include large tumor size, lymphovascular space invasion (LVSI) and deep cervical interstitial infiltration [5-6]. High-risk factors include lymph node metastasis (LNM), parametrial invasion and resection margin involvement [7-8]. The existence of risk factors is associated with higher recurrence rate and poor survival outcome in patients with early cervical cancer. These patients can benefit from postoperative RT or CCRT, prolong disease progression-free survival (PFS) time and overall survival (OS) time [7].

Considering the short survival time of patients with recurrent cervical cancer, it is vital to identify 
the prognostic factors for recurrent cervical cancer after initial treatment. However, the clinical features and the effect of each risk factor on the recurrent cervical cancer patients is much less known. In addition, according to the latest FIGO staging system, LNM is defined as stage IIIC, and the prognosis of these patients after radical surgery and adjuvant RT/CCRT is not very clear.

Therefore, the purpose of this retrospective study was to identify the recurrence pattern and prognostic factors of patients with recurrent cervical cancer after initial treatment with RHND followed by adjuvant chemoradiotherapy (RT/CCRT) .

\section{Patients and methods}

\section{Study population}

Medical records of recurrent cervical cancer patients who initially treated with RHND and adjuvant chemoradiotherapy (RT/CCRT) registered from January 2012 to May 2018 in Liaoning Cancer Hospital were retrospectively analyzed.

Inclusion criteria included histologically diagnosed cervical cancer, preoperative FIGO stage IB-IIA disease, no history of neoadjuvant chemoradiotherapy, received RHND, received postoperative pelvic radiotherapy (dose $\geq 40 \mathrm{~Gy}$ ) with or without concurrent chemotherapy.

Pre-treatment examinations included gynecological examination, blood routine examination, blood biochemical examination, urine routine examination, squamous cell carcinoma antigen ( $\mathrm{SccAg}$ ), chest and abdomen CT, pelvic magnetic resonance imaging (MRI) or PET/CT. Cystoscopy and colonoscopy were performed when bladder and rectum were suspected to be involved. The pathological reports included histological subtype, pathological differentiation degree, tumor size, LVSI, interstitial infiltration depth, number of lymph nodes dissected, number of positive lymph nodes in each site, parametrial invasion and margin resection involvement were analyzed retrospectively.

\section{Treatment}

All patients initially received adjuvant RT or CCRT after RHND. Conformal radiotherapy (CRT) or intensity-modulated radiotherapy (IMRT) started within 4-6 weeks after radical surgery. According to the CTV guidelines of radiotherapy tumor group for whole pelvis, the clinical target volume (CTV) included parauterine area, upper vagina and pelvic lymph drainage area (common iliac blood vessel area, internal and external iliac blood vessel area, obturator lymph node area and presacral lymph node area) . In addition, the para-aortic lymph node area was included when para-aortic lymph node metastasis occurred.

A dose of 44.0-50.4Gy in 22-28 fractions (1.8-2.0Gy/day) was delivered to at least $95 \%$ of the PCTV. Patients received radiotherapy 5 fractions per week over 4.5-6 weeks. Intracavitary brachytherapy was used for patients with vaginal lesions close to the resection margin $(\leq 5 \mathrm{~mm})$ or with positive resection margin. The total dose was 10-18Gy in 2-6 fractions. Dose limits for organs at risk were as follows: spinal cord D0.1 cc $\leq 45 \mathrm{gy}$, small intestine D2cc $\leq 54 \mathrm{gy}$, bladder D50\% $\leq 45 \mathrm{gy}$, rectum D50\% $\leq 45 \mathrm{gy}$.

The concurrent chemotherapy based on cisplatin included weekly cisplatin $\left(30-40 \mathrm{mg} / \mathrm{m}^{2}\right)$ for $4-6$ courses or 3-4 courses of paclitaxel and cisplatin / carboplatin every 3 weeks.

Treatment of recurrent cervical cancer depends on the initial treatment methods, site of recurrence and patient's physical condition. The treatment modes included operation, radiotherapy, chemotherapy, comprehensive treatment, immunotherapy and palliative care. Surgery was defined as therapeutic 
surgery, not including symptomatic surgery. Palliative care was defined as symptomatic supportive care.

\section{Follow-up evaluations}

Patients received first follow-up evaluation one month after the end of treatment. Then, every 3 months in the first 2 years, every 6 months in the 3-5 years, and once a year after 5 years. Main follow-up examinations included gynecological examination, SccAg, chest and abdomen CT and pelvic MRI. PET-CT was recommend only when disease relapse was suspected.

Local recurrence (LR) was defined as any disease relapse in the radiation field, including vaginal stump and pelvic lymph node area below aortic bifurcation. Distant metastasis (DM) was defined as disease relapse outside the radiation field. Disease PFS was defined as time from the day of surgery to disease relapse or the latest follow-up. OS after recurrence was defined as time from the day of disease relapse was diagnosed to cervical cancer-specific death or the latest follow-up. In our study, death after recurrence was defined as specific death of cervical cancer, excluding death due to other reasons.

\section{Statistical analysis}

The risk factors for specific death of recurrent cervical cancer were analyzed. The variables included age (continuous variable), disease early relapse (recurrence occurred within 6 months after the day of surgery), number of recurrent organs, recurrence site, histological diagnosis, tumor size, pathological differentiation degree, LVSI, interstitial infiltration depth, parametrial invasion, resection margin involvement, pelvic LNM, para-aortic LNM were categorical variables.

Chi-square test or Fisher exact test were used for univariate analysis of categorical variables. Variables with statistical significance in univariate analysis were used in the subsequent multivariate analysis. Multivariate analysis was performed with Cox proportional hazard model. Kaplan-Meier method and Log-rank test were used to evaluate the influence of risk factors on the survival rate after disease relapse. Results showed as risk ratio (HR) of $95 \%$ confidence interval. $\mathrm{P}<0.05$ was considered as statistically significant.

\section{Results}

\section{Patient characteristics}

Between January 2012 and May 2018, a total of 415 patients with biopsy proven cervical cancer were treated with RHND followed by adjuvant radiochemotherapy (RT/CCRT). 153 patients occurred disease relapse and were enrolled in our study.

The clinicopathological characteristics of recurrent cervical cancer patients was presented in table 1. The median age was 47 years old (range: 29-67 years). The main histological type was squamous cell carcinoma $(92.8 \%, 142 / 153)$. Deep interstitial infiltration was the most common risk factors $(93.5 \%$, 143/153), followed by LVSI (77.1\%, 118/153) and LNM (43.8\%, 67/153). 21 patients had positive resection margin $(13.7 \%, 21 / 153)$. Among 153 patients, 82 patients had high-risk factors, 76 of them had single high-risk factor, and the remaining 6 patients had two high-risk factors. 131 patients received adjuvant concurrent chemoradiotherapy (RT/CCRT) after surgery, and 22 patients received simple radiotherapy. 
Table 1 Basic characteristics of recurrent cervical cancer patients after initial treatment

\begin{tabular}{|c|c|c|}
\hline & Number & Proportion $(\%)$ \\
\hline \multicolumn{3}{|l|}{ Age (years old) } \\
\hline$\leq 50$ & 96 & 62.7 \\
\hline$>50$ & 57 & 37.3 \\
\hline \multicolumn{3}{|l|}{ Postoperative FIGO } \\
\hline \multicolumn{3}{|l|}{ staging (2018) } \\
\hline IA & 0 & 0 \\
\hline IB & 59 & 38.6 \\
\hline IIA & 26 & 17.0 \\
\hline IIIC1 & 45 & 29.4 \\
\hline IIIC2 & 23 & 15.0 \\
\hline \multicolumn{3}{|l|}{ Histological subtype } \\
\hline Squamous cell carcinoma & 142 & 92.8 \\
\hline Adenocarcinoma & 9 & 5.9 \\
\hline $\begin{array}{l}\text { Adenosquamous } \\
\text { carcinoma }\end{array}$ & 2 & 1.3 \\
\hline \multicolumn{3}{|l|}{ Lesion diameter } \\
\hline$<4 \mathrm{~cm}$ & 94 & 61.4 \\
\hline$\geq 4 \mathrm{~cm}$ & 59 & 38.6 \\
\hline \multirow{2}{*}{\multicolumn{3}{|c|}{$\begin{array}{l}\text { Pathological differentiation } \\
\text { degree }\end{array}$}} \\
\hline & & \\
\hline High/High-medium & 34 & 22.2 \\
\hline Medium & 52 & 34.0 \\
\hline Low / Medium-Low & 67 & 43.8 \\
\hline \multicolumn{3}{|l|}{ Interstitial infiltration depth } \\
\hline$<1 / 2$ & 10 & 6.5 \\
\hline$\geq 1 / 2$ & 143 & 93.5 \\
\hline \multicolumn{3}{|c|}{ Lymphovascular space invasion } \\
\hline Yes & 118 & 77.1 \\
\hline No & 35 & 22.9 \\
\hline \multicolumn{3}{|l|}{ Parametrial infiltration } \\
\hline Yes & 0 & 0 \\
\hline No & 153 & 100 \\
\hline \multicolumn{3}{|l|}{ Resection margin involvement } \\
\hline Yes & 21 & 13.7 \\
\hline No & 132 & 86.3 \\
\hline \multicolumn{3}{|l|}{ Lymph node metastasis } \\
\hline Yes & 67 & 43.8 \\
\hline No & 86 & 56.2 \\
\hline
\end{tabular}

\section{Recurrence pattern}

In table 2, we report the patterns of relapse and treatment mode of recurrent cervical cancer patients. Totally 153 patients had disease relapse in our study. 105 patients $(105 / 153,68.6 \%)$ had distant 
metastasis (DM), of which 67 (67/153, 43.8\%) had simple DM, and 38 (38/153, 24.8\%) had DM with local relapse (LR). 48 (48/153, 31.4\%) had simple LR.

Lung was the most common distant metastasis site $(55 / 105,52.4 \%)$, followed by distant lymph node $(44 / 105,41.9 \%)$ and bone metastasis $(34 / 105,32.4 \%)$. Liver metastasis accounted for 19.0\% (20/105), and other sites accounted for 8.6\% (9/105). 43.8\% (46/105) of patients with DM had multiple organ recurrence.

Our study showed that the median disease PFS time was 16 months (range: 5-70 months), 75.2\% $(115 / 153)$ patients had recurrence within 2 years, and 24.8\% (38/153) had early disease relapse (recurrence occurred within 6 months after the day of surgery).

The treatment after disease relapse included simple palliative chemotherapy in 73 cases (47.7\%), simple palliative radiotherapy in 3 cases $(2 \%)$, simple surgery in 7 cases $(4.6 \%)$, chemotherapy combined with radiotherapy in 35 cases $(22.9 \%)$, surgery combined with chemotherapy/radiotherapy in 19 cases $(12.4 \%)$, chemotherapy combined with targeted therapy in 5 cases $(3.3 \%)$, immunotherapy in 3 cases $(2 \%)$, and palliative care in 8 cases $(5.1 \%)$.

Table 2 Recurrence patterns and treatment mode of recurrent cervical cancer patients

\begin{tabular}{|c|c|c|}
\hline & Patients No. & Proportion $(\%)$ \\
\hline \multicolumn{3}{|l|}{ Disease PFS (month) } \\
\hline$\leq 6$ & 38 & 24.8 \\
\hline$>6$ & 115 & 75.2 \\
\hline \multicolumn{3}{|l|}{ Recurrence site } \\
\hline LR & 48 & 31.4 \\
\hline $\mathrm{DM}$ & 67 & 43.8 \\
\hline $\mathrm{LR}+\mathrm{DM}$ & 38 & 24.8 \\
\hline \multicolumn{3}{|l|}{ Number of recurrent organs } \\
\hline Single & 84 & 54.9 \\
\hline Multiple & 69 & 45.1 \\
\hline \multicolumn{3}{|l|}{ Treatment after recurrence } \\
\hline Chemotherapy & 73 & 47.7 \\
\hline Radiotherapy & 3 & 2.0 \\
\hline Surgery & 7 & 4.6 \\
\hline Chemoradiotherapy & 35 & 22.9 \\
\hline $\begin{array}{l}\text { Surgery+chemotherapy/chem } \\
\text { oradiotherapy }\end{array}$ & 19 & 12.4 \\
\hline $\begin{array}{l}\text { Chemotherapy+targeted } \\
\text { therapy }\end{array}$ & 5 & 3.3 \\
\hline Immunotherapy & 3 & 2.0 \\
\hline Palliative therapy & 8 & 5.1 \\
\hline
\end{tabular}

\section{Survival analysis}

Our study further analyzed the survival outcome of cervical cancer patients after recurrence. The median follow-up time was 19 months (5-55 months). The median survival time after disease relapse were 26 months (range: 10-54 months) and 18 months (range: 5-55 months) in patients with simple LR and simple DM, respectively. The median survival time of patients with LR+DM was 17.5 months 
(range: 5-33 months).

The survival curves according to the number of organ involvement and the recurrence patterns are shown in Fig. 1-2. 69 patients were diagnosed as multi-organ involvement. The 1 -, 2 -, and 3-year survival rates were $47.06 \%, 14.71 \%$ and $2.9 \%$, respectively. Our study indicated that the survival of patients with recurrences in multiple organs was signifificantly lower in comparison to those with recurrences in single organ $(P<0.001)$. In addition, statistical analysis showed that there were significant difference in survival rate between different recurrence patterns $(P<0.001)$. The survival rate of patients with simple DM and LR+DM was significantly lower than that of patients with simple LR $(P=0.006, P<0.001)$. Furthermore, the survival rate of patients with $\mathrm{LR}+\mathrm{DM}$ was significantly lower than that of patients with simple DM $(P=0.046)$.

Fig. 1 Overall survival comparing single organ and multiple organs

Fig.2 Overall survival comparing different recurrence patterns

Table 3 and table 4 present the detailed information of univariate and multivariate analysis . Univariate analysis showed that disease stage, tumor size, pathological differentiation degree, positive resection margin, positive lymph nodes in pelvic cavity, positive para-aortic lymph nodes, different metastatic sites, number of involved organs, treatment modes after disease relapse and early disease recurrence were associated with death after disease relapse. In multivariate analysis, the variables with statistical differences in the above univariate analysis were included in the Cox proportional hazard regression model. The results showed that the risk factors of death after disease relapse were positive resection margin, positive common iliac lymph node, positive para-aortic lymph node, different metastatic sites, no treatment after recurrence, and early disease recurrence.

Table 3 Univariate analysis for factors associated with survival outcomes in patients with recurrent cervical cancer

\begin{tabular}{llc}
\hline Variable & $\begin{array}{l}\text { Median Survival } \\
\text { Time }\end{array}$ & $P$ value \\
\hline Age (years old) & & 0.459 \\
$\quad \leq 50$ & 19 & \\
$\quad>50$ & 20 & \\
Postoperative FIGO staging & & 0.000 \\
$(2018)$ & & \\
$\quad$ IB & 22 & \\
IIA & 25.5 & \\
IIIC1 & 18 & \\
IIIC2 & 11 & 0.045 \\
Tumor size (cm) & & \\
$\quad<4$ & 20.5 & \\
$\quad \geq 4$ & 17 & 0.570 \\
Histology & &
\end{tabular}




Squamous cell carcinoma
Non-squamous cell carcinoma
Pathological differentiation
degree
Low/Medium-Low
Medium
High/High-medium
Lymphovascular space invasion
Yes
No
Interstitial infiltration depth
$<1 / 2$
$\geq 1 / 2$

Resection margin involvement 0.012

Yes

Lymph node metastasis

No

Obturator LNM

0.000

Yes

No

Internal and external iliac LNM 0.000 Yes

No

22

Common iliac LNM

Yes

No

Para-aortic LNM Yes

Recurrence site

Simple LR

Number of recurrent organs

Recurrence time (month)

Treatment mode after disease relapse 


\begin{tabular}{ll} 
Chemotherapy & 18 \\
Chemoradiotherapy & 25 \\
Surgery+chemotherapy & 19.5 \\
Refuse treatment & 10.5 \\
\hline
\end{tabular}

Table 4 Cox proportional hazard analysis for prognositc factors associated with survival outcomes in recurrent cervical cancer

\begin{tabular}{|c|c|c|c|}
\hline \multirow{2}{*}{ Variable } & \multicolumn{3}{|c|}{ Overall survival } \\
\hline & HR & $95 \% \mathrm{CI}$ & $P$ value \\
\hline Resection margin involvement & & & 0.002 \\
\hline No & Reference & & \\
\hline Yes & 2.728 & $1.455-5.115$ & \\
\hline Common iliac LNM & & & 0.000 \\
\hline No & Reference & & \\
\hline Yes & 4.516 & $2.702-7.546$ & \\
\hline Para-aortic LNM & & & 0.023 \\
\hline No & Reference & & \\
\hline Yes & 1.900 & $1.092-3.307$ & \\
\hline Recurrence site & & & 0.002 \\
\hline Simple LR & Reference & & \\
\hline Simple DM & 2.054 & $1.054-4.006$ & 0.035 \\
\hline $\mathrm{LR}+\mathrm{DM}$ & 3.895 & $1.846-8.217$ & 0.000 \\
\hline Recurrence time $\leq 6$ months & & & 0.012 \\
\hline No & Reference & & \\
\hline Yes & 2.148 & $1.185-3.894$ & \\
\hline Treatment mode after disease relapse & & & 0.032 \\
\hline Simply chemotherapy & Reference & & \\
\hline Chemoradiotherapy & 0.716 & $0.418-1.228$ & 0.225 \\
\hline Surgery+chemotherapy & 0.487 & $0.212-1.119$ & 0.090 \\
\hline Refuse treatment & 2.635 & $1.134-6.121$ & 0.024 \\
\hline
\end{tabular}

\section{Discussion}

Palliative chemotherapy is the main treatment for patients with recurrent cervical cancer. Individualized treatment also includes surgery, radiotherapy, targeted therapy and immunotherapy, etc. Although great efforts have been made to prolong the survival time of patients with recurrent cervical cancer in the past decades, the prognosis of these patients is still not optimistic. Some studies reported that the 1-year survival rate of recurrent cervical cancer was only $15 \%-20 \%$ [9].

This study analyzed the recurrence patterns and prognostic factors of patients with recurrent cervical cancer after radical surgery followed by adjuvant chemoradiotherapy (RT/CCRT). Most patients (75.2\%) had disease relapse within 2 years. The median disease PFS was 16 months, which was 
consistent with the data reported in earlier studies [10-13]. 24.8\% patients were early disease recurrence in this study. In addition, our study showed that early disease recurrence was an independent risk factor for the prognosis of patients with recurrent cervical cancer $(\mathrm{P}=0.012)$. NCCN guidelines suggest that follow-up evaluation should be conducted every 3 months within 2 years after initial treatment completed. Early detection and early treatment may improve the prognosis of patients with early disease recurrence. Therefore, cervical cancer patients with high risk factors proved by postoperative pathology can consider increasing the frequency of reexamination within half a year after the end of initial treatment.

Our study indicated that the surgical variables of the independent risk factors leading to poor survival of recurrent cervical cancer included positive resection margin, positive common iliac lymph node and positive para-aortic lymph node, which were all high risk factors in pathological examination. Cisplatin based concurrent chemotherapy during radiotherapy is the standard treatment of postoperative adjuvant therapy for middle and high-risk cervical cancer patients. Additional treatment could be considered to prolong the survival time of patients with pathological high-risk factors, such as targeted therapy, systemic chemotherapy before/after radiotherapy. In another study [14], 34 cervical cancer patients with stage IIB-IIIB were treated with consolidation chemotherapy (paclitaxel combined with nedaplatin) for 4 cycles. The results showed that the complete remission rate was $88 \%$, and the 2 -year disease PFS rate was $82 \%$. Our study suggested that the concept of consolidation therapy may be feasible for cervical cancer patients with postoperative adjuvant chemoradiotherapy if there are high-risk factors in surgical specimens.

In recent years, many studies have investigated the effect of positive pelvic lymph nodes on the survival outcome of cervical cancer. Shyu et al. [15] showed that 5-year survival rate of recurrent cervical cancer patients who underwent radical surgery with LNM was significantly lower than that of patients with no LNM. Meir et al. [16] suggested that LNM is an independent risk factor for OS and disease PFS of recurrent cervical cancer. The latest FIGO staging system classifies LNM as IIIC stage, which indicates that LNM can lead to worse prognosis. Pelvic lymph nodes metastasis were classified as stage IIIC1 in generalities according to the 2018 FIGO staging system, however, there was no more detailed staging. Our study found that positive common iliac lymph node was independent risk factor for poor prognosis in recurrent cervical cancer patients, while other sites of pelvic lymph nodes metastasis are not. Therefore, further study are necessary to clarify the difference between the prognosis of patients with positive common iliac lymph nodes and those with positive internal iliac, external iliac and obturator lymph nodes.

Our study indicated that the independent risk factors for poor survivals after disease relpase of cervical cancer patients also included distant metastasis (simple DM / LR+DM) and multiple organs involvement. This is consistent with previous studies. Qiu et al. [17] evaluated the prognosis of 121 patients with recurrent cervical cancer after radical surgery and indicated that DM was significantly associated with poor prognosis. Moreover, we found that the incidence of LVSI was higher in patients with recurrent cervical cancer. It may be related to the fact that LVSI is more likely to occur hematogenous metastasis to distant organs [18]. Systemic chemotherapy as consolidation therapy in patients with LVSI may reduce distant metastasis incidence.

This study is a single-institution retrospective study, which may lead to selection bias and time-trend bias. In addition, sample size is also a limitation. 


\section{Conclusion}

Our study indicated that positive resection margin, positive common iliac lymph node and para-aortic lymph node, no treatment after disease recurrence, early disease relapse and DM were significantly associated with poor prognosis of patients with recurrent cervical cancer.

Patients with high-risk factors and LVSI could be considered to receive targeted therapy, consolidation chemotherapy or oral drug maintenance therapy after adjuvant chemoradiotherapy to reduce disease relapse and prolong survival time. Patients can increase the frequency of reexamination within half a year after the end of initial treatment, which can help the patients with early disease recurrence receive treatment earlier and improve the prognosis.

Ethics approval Ethical approval was waived by the local Ethics Committee of the Hospital in view of the retrospective nature of the study and all the procedures being performed were part of the routine care.

Funding details No funding was obtained for this study.

Conflict of interest All the authors declare no conflict of interest.

Informed Consent Informed consent was obtained from all individual participants included in the study.

\section{References}

1. Jemal A, Bray F, Center MM, Ferlay J, Ward E, Forman D (2011) Global cancer statistics. CA Cancer J Clin 61(2):69-90.

2. Bray F, Ferlay J, Soerjomataram I, Siegel RL, Torre LA, Jemal A (2018) Global cancer statistics 2018: GLOBOCAN estimates of incidence and mortality worldwide for 36 cancers in 185 countries.

CA Cancer J Clin 68(6):394-424.

3. Waggoner SE. Cervical cancer (2003) Lancet 361(9376):2217-25.

4. Yang K, Park W, Huh SJ, Bae DS, Kim BG, Lee JW (2017) Clinical outcomes in patients treated with radiotherapy after surgery for cervical cancer. Radiat Oncol J 35(1):39-47.

5. Delgado G, Bundy B, Zaino R, Sevin BU, Creasman WT, Major F (1990) Prospective surgical-pathological study of disease-free interval in patients with stage IB squamous cell carcinoma of the cervix: a Gynecologic Oncology Group study. Gynecol Oncol 38(3):352-7.

6. Sedlis A, Bundy BN, Rotman MZ, Lentz SS, Muderspach LI, Zaino RJ (1999) A randomized trial of pelvic radiation therapy versus no further therapy in selected patients with stage IB carcinoma of the cervix after radical hysterectomy and pelvic lymphadenectomy: A Gynecologic Oncology Group Study. Gynecol Oncol 73(2):177-83.

7. Peters WA 3rd, Liu PY, Barrett RJ 2nd, Stock RJ, Monk BJ, Berek JS, Souhami L, Grigsby P, Gordon W Jr, Alberts DS (2000) Concurrent chemotherapy and pelvic radiation therapy compared with 
pelvic radiation therapy alone as adjuvant therapy after radical surgery in high-risk early-stage cancer of the cervix. J Clin Oncol 18(8):1606-13.

8. Kim D, Ki Y, Kim W, Park D, Lee J, Lee J, Jeon H, Nam J (2018) Adjuvant external beam radiation and brachytherapy for vaginal resection margin positive cervical cancer. Radiat Oncol J 36(2):147-152.

9. Benedet JL, Odicino F, Maisonneuve P, Beller U, Creasman WT, Heintz AP, Ngan HY, Pecorelli S (2003) Carcinoma of the cervix uteri. Int J Gynaecol Obstet Suppl 1:41-78.

10. Wang CJ, Lai CH, Huang HJ, Hong JH, Chou HH, Huang KG, Lin JD (1999) Recurrent cervical carcinoma after primary radical surgery. Am J Obstet Gynecol 181(3):518-24.

11. Qiu JT, Abdullah NA, Chou HH, Lin CT, Jung SM, Wang CC, Chen MY, Huang KG, Chang TC, Lai CH (2012) Outcomes and prognosis of patients with recurrent cervical cancer after radical hysterectomy. Gynecol Oncol 127(3):472-7.

12. Samlal RA, Van Der Velden J, Van Eerden T, Schilthuis MS, Gonzalez Gonzalez D, Lammes FB (1998) Recurrent cervical carcinoma after radical hysterectomy: an analysis of clinical aspects and prognosis. Int J Gynecol Cancer 8(1):78-84.

13. Ansink A, de Barros Lopes A, Naik R, Monaghan JM (1996) Recurrent stage IB cervical carcinoma: evaluation of the effectiveness of routine follow up surveillance. Br J Obstet Gynaecol 103(11):1156-8.

14. Zhang MQ, Liu SP, Wang XE (2010) Concurrent chemoradiotherapy with paclitaxel and nedaplatin followed by consolidation chemotherapy in locally advanced squamous cell carcinoma of the uterine cervix: preliminary results of a phase II study. Int J Radiat Oncol Biol Phys 78(3):821-7. 15. Ng HT, Kan YY, Chao KC, Yuan CC, Shyu SK (1987) The outcome of the patients with recurrent cervical carcinoma in terms of lymph node metastasis and treatment. Gynecol Oncol 26(3):355-63. 16. Joo JH, Kim YS, Nam JH (2018) Prognostic significance of lymph node ratio in node-positive cervical cancer patients. Medicine (Baltimore) 97(30):e11711.

17. Qiu JT, Abdullah NA, Chou HH, Lin CT, Jung SM, Wang CC, Chen MY, Huang KG, Chang TC, Lai CH (2012) Outcomes and prognosis of patients with recurrent cervical cancer after radical hysterectomy. Gynecol Oncol 127(3):472-7.

18. Viswanathan AN, Deavers MT, Jhingran A, Ramirez PT, Levenback C, Eifel PJ (2004) Small cell neuroendocrine carcinoma of the cervix: outcome and patterns of recurrence. Gynecol Oncol 93(1):27-33. 
Figures

Fig.1

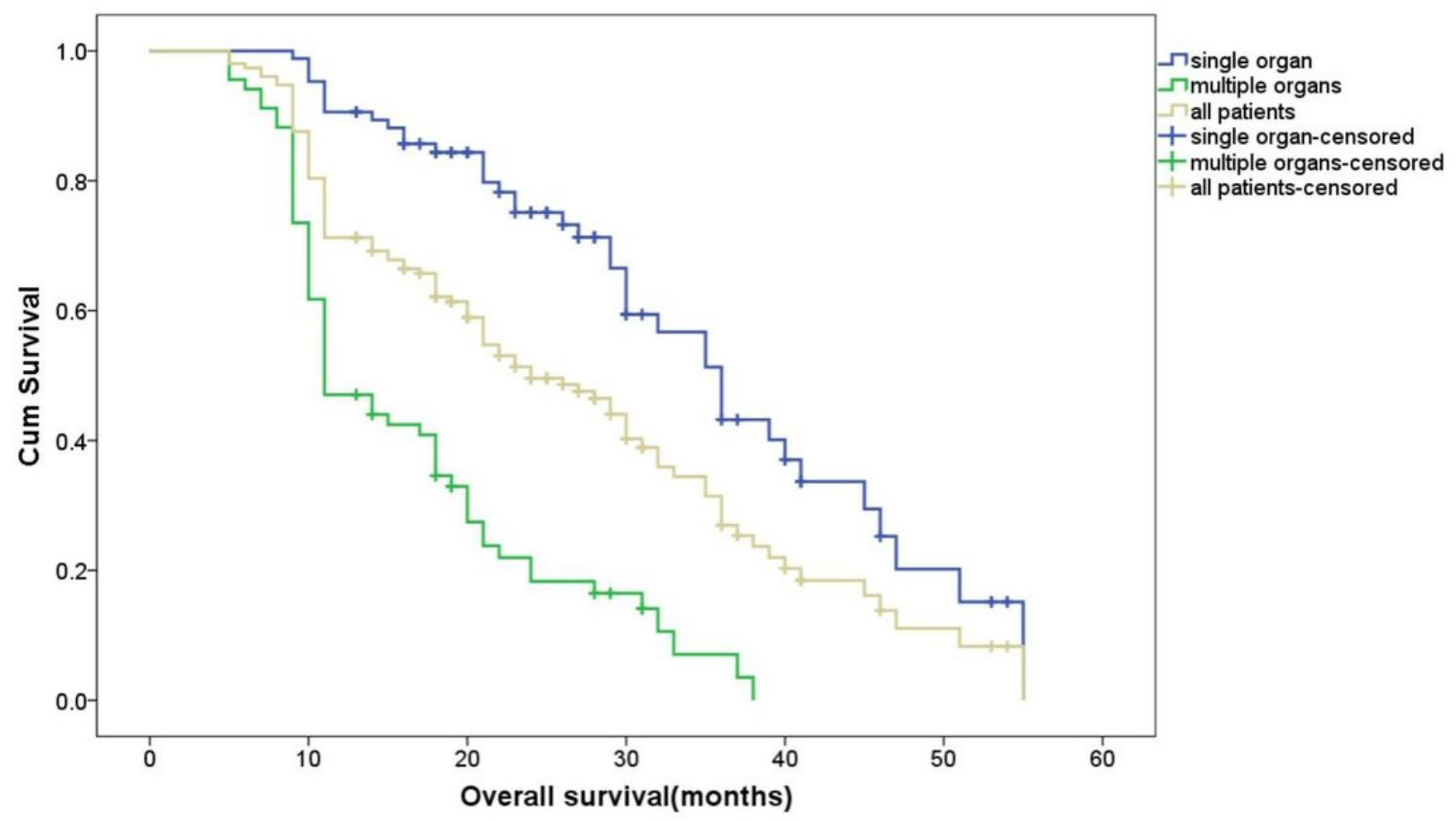

Figure 1

Overall survival comparing single organ and multiple organs 
Fig. 2

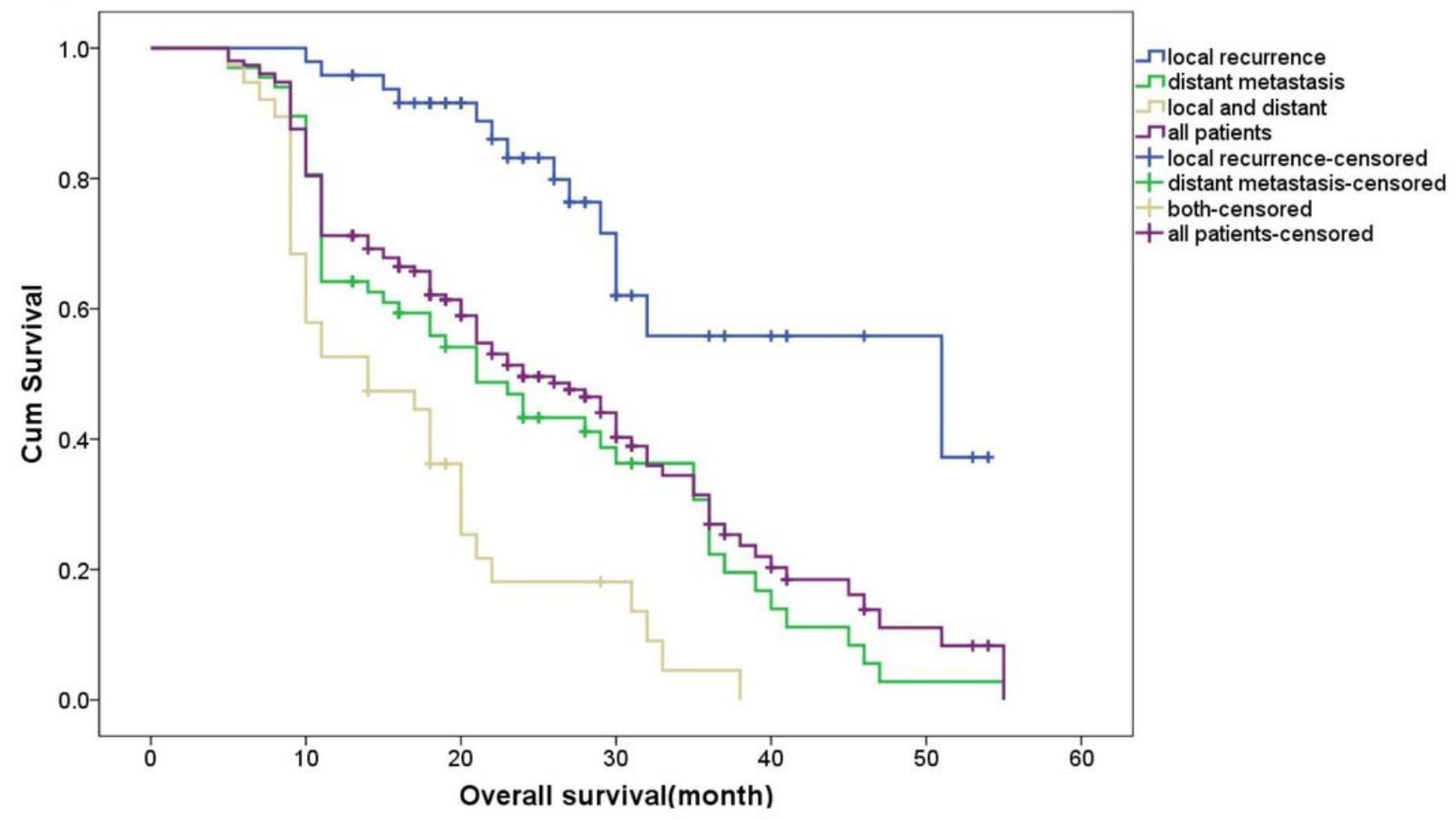

Figure 2

Overall survival comparing different recurrence patterns 